\title{
Extended function point analysis prototype: with security costing estimation
}

\begin{abstract}
Software cost estimation is a complex activity that requires knowledge of a number of key attributes about the project for which the estimate is being constructed. Nevertheless, security is becoming more and more important in most of the software construct. Reviews on several widely recognized parametric estimation models found the insufficient of current estimation in software security costing. Comparison of common security standards successfully highlighted software security characteristics to be considered in cost estimation. A survey based on the rating of these characteristics has been conducted. Analysis results from RASCH have validated the relative importance of these characteristics based on Multimedia Super Corridor (MSC) software developers perspective. These validated characteristics are then extended the calculation and implemented in a Function Point Analysis prototype. The paper also points to the steps of calibrating the calculation through this prototype by conducting controlled experiments.
\end{abstract}

Keyword: Software cost estimation; Security costing; RASCH analysis method; Function point analysis; Software security characteristics 